\title{
New Conversion Laws for CO Observations
}

\author{
Peter J. Barnes ${ }^{1,2}$, Erik Muller ${ }^{3}$, Audra K. Hernandez ${ }^{4}$ \\ Ana Duarte-Cabral ${ }^{5}$ and Frederic Schuller ${ }^{6}$
}

\footnotetext{
${ }^{1}$ Astronomy Dept., University of Florida, PO Box 112055, Gainesville FL 32611, USA

${ }^{2}$ School of Science \& Technology, University of New England, Armidale NSW 2351, Australia

${ }^{3}$ National Astronomical Observatory of Japan, Chile Observatory, Tokyo 181-8588, Japan

${ }^{4}$ Astronomy Dept., University of Wisconsin, 475 North Charter St., Madison, WI 53706, USA

${ }^{5}$ School of Physics, University of Exeter, Stocker Road, Exeter, EX4 4QL, United Kingdom

${ }^{6}$ Max-Planck-Institut für Radioastronomie, Auf dem Hügel 69, 53121, Bonn, Germany email: pjb@ufl.edu, erik.muller@nao.ac.jp, hernande@astro.wisc.edu
}

\begin{abstract}
We describe new conversion laws, from CO molecular line data to inferred mass column, based on observations of the three main CO isotopologues in several surveys of the Galactic Plane. The new conversion laws replace the use of the single " $X$-factor" in widespread use, with a more physically-based relationship between the CO line's optical depth, excitation, and column density. It has the effect of increasing the inferred mass column, over the single $X$-factor, by typically a factor of $2-3$. This means that the molecular mass of the Milky Way may have been substantially underestimated in previous studies, and suggests that scaling laws like the Kennicutt-Schmidt relations may also need to be recalibrated. Because of its statistical basis on a large fraction of our Galaxy's ISM, this new law is also recommended for use in studies of other Milky-Way-analogue spiral galaxies.
\end{abstract}

Keywords. galaxies: abundances, ISM: molecules, astrochemistry

\section{The Milky Way as a Near-Field Extragalactic Calibrator}

Recent large-scale, multi-species surveys of molecular emission in the Galactic Plane, such as CHaMP, ThrUMMS, SEDIGISM and others (see below), have found strong line ratio variations among many species, both spatially and in velocity across the Milky Way, even for the 3 main $\mathrm{CO}$ isotopologues. These indicate different opacity and excitation environments for the molecular emission, and enable a spatially- and velocity-resolved calculation of the column density in such species, with fewer assumptions than has traditionally been the case. The (sub-)parsec scale resolution and high sensitivity of these surveys (resolving the formation scale of individual clusters), across many $\mathrm{kpc}$ of the Galactic disk, provide "ground truth" for calibrating extragalactic conversion laws.

\section{The Survey Projects}

1. CHaMP @ Mopra: $37^{\prime \prime}$ resolution, $300^{\circ}>l>280^{\circ}, J=1 \rightarrow 0$ lines of $\mathrm{HCO}^{+}, \mathrm{HCN}$, $\mathrm{N}_{2} \mathrm{H}^{+}$, iso-CO, CN, 25 others; Barnes et al. (2011), Barnes et al. (2013), Barnes et al. (2016), Schap et al. (2016).

2. ThrUMMS @ Mopra: $72^{\prime \prime}$ resolution, $360^{\circ}>l>300^{\circ}, J=1 \rightarrow 0$ lines of iso-CO, CN; Barnes et al. (2015).

3. California Cloud @ SMT: $65^{\prime \prime}$ resolution, $J=1 \rightarrow 0$ lines of iso-CO; Kong et al. (2015). 4. SEDIGISM @ APEX: $30^{\prime \prime}$ resolution, $l=18^{\circ}-360^{\circ}-300^{\circ}, J=2 \rightarrow 1$ lines of ${ }^{13} \mathrm{CO}, \mathrm{C}^{18} \mathrm{O}$; Schuller et al. (2016).

\section{Radiative Transfer Analysis}

Among CHaMP, ThrUMMS, and SEDIGISM we see dramatic variations in the CO line ratios, indicating a very wide range of optical depth and excitation conditions, from warm and translucent to cold and opaque. Assuming only (1) plane-parallel LTE, (2) a common $T_{\text {ex }}$ among the 3 isotopologues, and (3) a fixed abundance ratio $R_{13}=\left[{ }^{12} \mathrm{CO}\right] /\left[{ }^{13} \mathrm{CO}\right]=$ 60 , we solved for the $T_{\mathrm{ex}}, \tau \mathrm{s}$, and $R_{18}=\left[{ }^{13} \mathrm{CO}\right] /\left[\mathrm{C}^{18} \mathrm{O}\right]$ in the ThrUMMS data (Barnes 


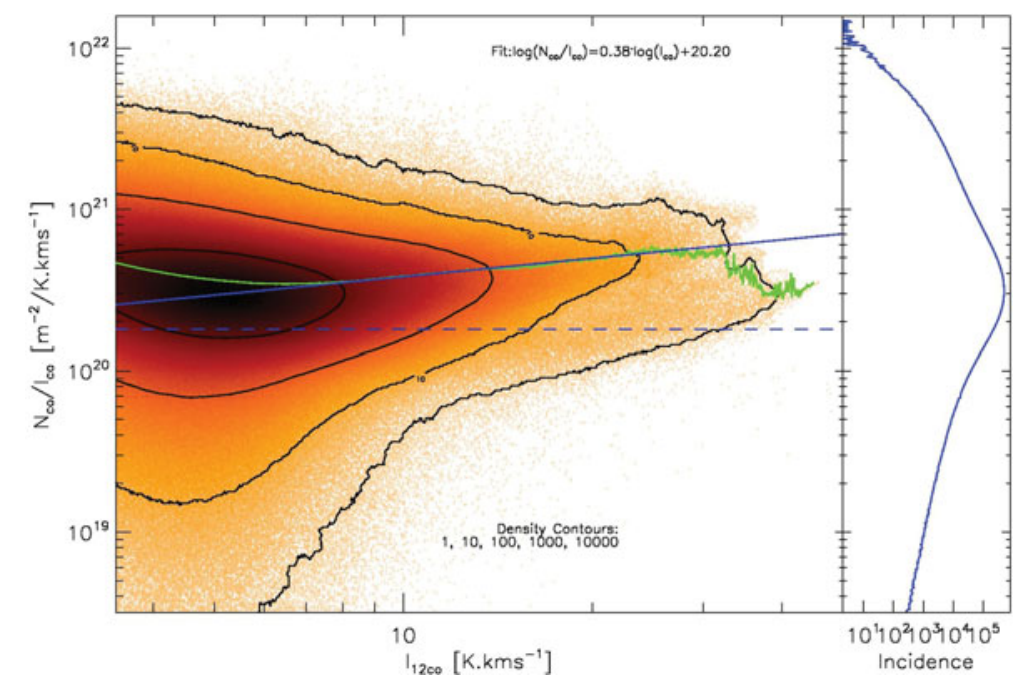

Figure 1. Ratio of CO column density $N$ to integrated intensity $I$, as a function of $I$, from ThrUMMS (Barnes et al. (2015)).

et al. (2015)), and then calculated the true CO column density across all $10^{9}$ voxels in the survey area. The population of cold clouds in particular have optical depths for ${ }^{12} \mathrm{CO}$ up to several 100s in some locations. We found that a constant $X$ factor converting $I_{\mathrm{CO}}$ to $N_{\mathrm{CO}}$ was not correct, but that the conversion followed a super-linear power law in $I$. A similar analysis of the ${ }^{13} \mathrm{CO} 2 \rightarrow 1 / 1 \rightarrow 0$ line ratio, using both ThrUMMS and SEDIGISM data, confirmed and extended this relationship. Although apparently different, the conversion laws' functional forms are a direct consequence of the different opacities in the two lines $\left(\sim 100\right.$ s for ${ }^{12} \mathrm{CO} J=1 \rightarrow 0, \sim$ a few for $\left.{ }^{13} \mathrm{CO} J=2 \rightarrow 1\right)$. All the conversion laws can be placed on a consistent footing, agreeing also with theoretical studies from (e.g.) Narayanan et al. (2012), which adds a scaling for metallicity.

\section{The New Conversion Laws}

We recommend the following conversion laws are applicable to disk galaxies in general, derived from the large-scale $\mathrm{CO}$ survey data as explained above:

$$
N_{H_{2}}=X_{\text {line }}\left(I(\mathrm{CO}) / \mathrm{K} \mathrm{km} \mathrm{s}^{-1}\right)^{p}\left(T_{e x} / 10 \mathrm{~K}\right)^{q}\left(Z / Z_{\odot}\right)^{-0.65}
$$

where $\quad X_{\text {line }}=1.8 \times 10^{24} \mathrm{~m}^{-2}, p=1.38, q=-0.7 \quad$ for "line" $={ }^{12} \mathrm{CO} \quad J=1 \rightarrow 0$

$$
X_{\text {line }}=1 \times 10^{25} \mathrm{~m}^{-2}, p=1, \quad q=0 \quad \text { for "line" }={ }^{13} \mathrm{CO} J=2 \rightarrow 1
$$

\section{Pending Applications}

We are engaged in several follow-up projects, including: comparison with dust continuum and HI surveys; deprojected 3D renderings of temperatures, densities, abundances, etc. throughout the molecular ISM; dependence of these on arm/interarm location, Galactocentric distance, environment; and several others.

\section{References}

Barnes, P. J., Yonekura, Y., Fukui, Y., et al. 2011, ApJS, 196, 12

Barnes, P. J., Ryder, S. D., O'Dougherty, S. N., et al. 2013, MNRAS, 432, 2231

Barnes, P. J., Muller, E., Indermuehle, B., et al. 2015, ApJ, 812, 6

Barnes, P. J., Hernandez, A. K., O'Dougherty, et al. 2016, ApJS, accepted

Kong, S., Lada, C. J., Lada, E. A., et al. 2015, ApJ, 805, 58

Narayanan, D., Krumholz, M. R., Ostriker, E. C., \& Hernquist, L. 2012, MNRAS, 421, 3127

Schap, W. J. III, Barnes, P. J., Ordonez, A., et al. 2016, MNRAS, subm.

Schuller, F., Csengeri, T., Urquhart, J. S., et al. 2016, A\&BA, subm. 